\title{
Multipotential stem cells recapitulate human infantile hemangioma in immunodeficient mice
}

\author{
Zia A. Khan, ${ }^{1}$ Elisa Boscolo, ${ }^{1}$ Arnaud Picard, ${ }^{1}$ Sarah Psutka, ${ }^{1}$ Juan M. Melero-Martin, ${ }^{1}$ \\ Tatianna C. Bartch, ${ }^{1}$ John B. Mulliken, ${ }^{2}$ and Joyce Bischoff ${ }^{1}$ \\ 1Vascular Biology Program and Department of Surgery, and 'Department of Plastic Surgery, Children's Hospital Boston, \\ Harvard Medical School, Boston, Massachusetts, USA.
}

\begin{abstract}
Infantile hemangioma is a benign endothelial tumor composed of disorganized blood vessels. It exhibits a unique life cycle of rapid postnatal growth followed by slow regression to a fibrofatty residuum. Here, we have reported the isolation of multipotential stem cells from hemangioma tissue that give rise to hemangioma-like lesions in immunodeficient mice. Cells were isolated based on expression of the stem cell marker CD133 and expanded from single cells as clonal populations. The CD133-selected cells generated human blood vessels 7 days after implantation in immunodeficient mice. Cell retrieval experiments showed the cells could again form vessels when transplanted into secondary recipients. The human vessels expressed GLUT-1 and merosin, immunodiagnostic markers for infantile hemangioma. Two months after implantation, the number of blood vessels diminished and human adipocytes became evident. Lentiviral expression of GFP was used to confirm that the hemangioma-derived cells formed the blood vessels and adipocytes in the immunodeficient mice. Thus, when transplanted into immunodeficient mice, hemangioma-derived cells recapitulated the unique evolution of infantile hemangioma - the formation of blood vessels followed by involution to fatty tissue. In summary, this study identifies a stem cell as the cellular origin of infantile hemangioma and describes for what we believe is the first time an animal model for this common tumor of infancy.
\end{abstract}

\section{Introduction}

Infantile hemangioma (IH) is a vascular tumor that occurs in $5 \%-10 \%$ of infants of mixed European descent (1). A hallmark of $\mathrm{IH}$ is its life cycle, which is divided into 3 stages. The proliferating phase spans the first year of postnatal life and is characterized by cellular masses of endothelium without a defined vascular architecture. The involuting phase begins around 1 year of age and continues for 3-5 years as blood vessels become more obvious and even enlarged. The involuted phase is reached by 5-8 years of age, at which point blood vessels are replaced with a fibrofatty residuum and capillary-sized channels. Intrinsic $(2,3)$ and extrin$\operatorname{sic}(4,5)$ factors have been hypothesized to contribute to the rapid growth and the slow, yet spontaneous regression. Nevertheless, the cellular origin of $\mathrm{IH}$ and the biologic signals for the uncontrolled growth remain elusive.

Histological analyses of IH in the early proliferating phase have generated a number of developmental theories suggesting an embryonic or primitive cell origin of IH. Virchow (6), Pack and Miller (7), and Malan (8) described IH as sequestered embryonic mesoderm and/or activated dormant angioblasts. The presence of CD34-positive cells in the interstitial compartment of IH led Smoller and Apfelberg to postulate a primitive vascular progenitor cell giving rise to both ECs and pericytes in the tumor (9). In

Nonstandard abbreviations used: BM-MSC, BM mesenchymal stromal cell; cbEPC, cord blood EPC; EBM-2, endothelial basal medium 2; EPC, endothelial progenitor cell; GFAP, glial fibrillar acidic protein; HDMEC, human dermal microvascular EC; HemEC, hemangioma EC; HemEPC, hemangioma EPC; HemSC, hemangiomaderived stem cell; IH, infantile hemangioma; MVD, microvessel density; NHDF, normal human dermal fibroblast; SC, stem cell.

Conflict of interest: The authors have declared that no conflict of interest exists. Citation for this article: J. Clin. Invest. 118:2592-2599 (2008). doi:10.1172/JCI33493. vitro studies have suggested that ECs isolated from IH have an immature fetal phenotype (10). We have shown that hemangioma ECs (HemECs) are clonal (3), indicating that the HemECs might originate from a single stem or progenitor cell. We also used human stem cell (SC) marker CD133 (also known as AC133 and prominin-1) (11) to detect (12) and isolate (13) hemangioma endothelial progenitor cells (HemEPCs). Our demonstration that HemECs and HemEPCs are more similar to normal human cord blood EPCs (cbEPCs) than mature human dermal microvascular ECs (HDMECs) (13) provides further support to the earlier histological studies that first suggested hemangioma is composed of immature vascular progenitor cells.

Herein we report on less-differentiated SCs in hemangioma specimens - referred to as hemangioma-derived SCs (HemSCs). The HemSCs exhibited robust proliferative and clonogenic capacity and differentiated into cells of multiple lineages. Importantly, clonal HemSC populations expanded in vitro from single cells produced human GLUT-1-positive microvessels in vivo. Over time, the human blood vessels diminished and human adipocytes became evident, reminiscent of the involuted phase of IH. In summary, our findings provide evidence for a SC origin of $\mathrm{IH}$, which is in contrast to the long-held view that IH arises from ECs (14).

\section{Results}

Isolation and characterization of HemSCs. Anti-CD133-coated magnetic beads have been used to isolate stem/progenitor cells from blood $(15)$ and from human tumors $(16,17)$. We isolated CD $133^{+}$cells from proliferating phase IH using this methodology and found that approximately $0.2 \%$ of the hemangioma cells were selected with anti-CD133-coated beads $(n=28$; age $11 \pm 1.47$ months, $\mathrm{CD}_{133}{ }^{+}$cells, $\left.0.2 \% \pm 0.027 \%\right)$. The CD133-selected cells, referred 
A
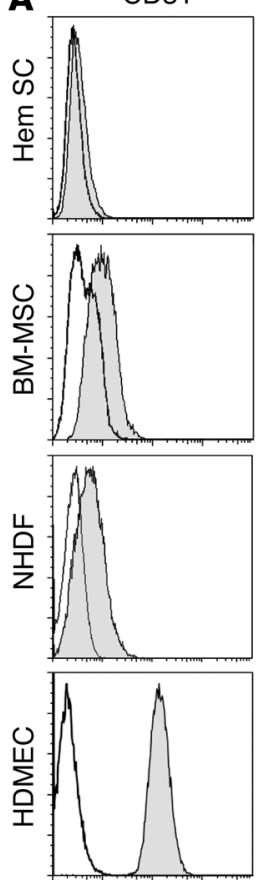

CD146
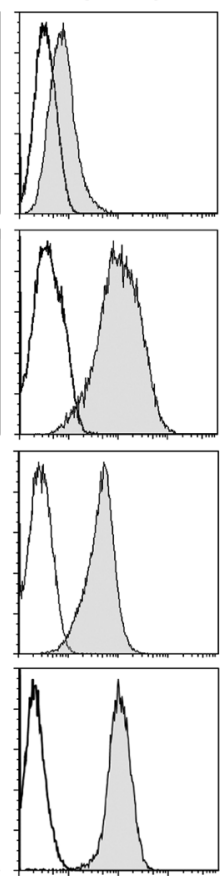

VEGF-R1
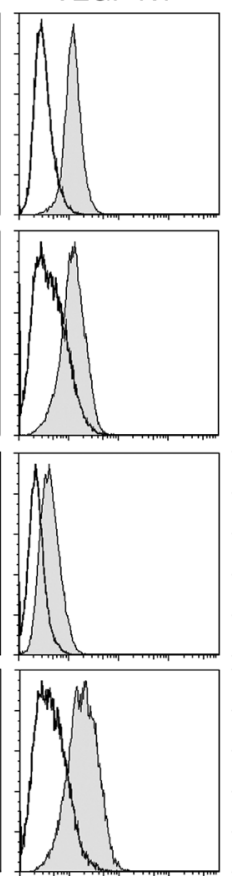

VEGF-R2
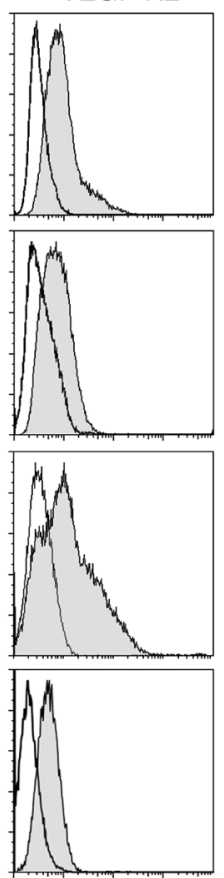

NRP-1
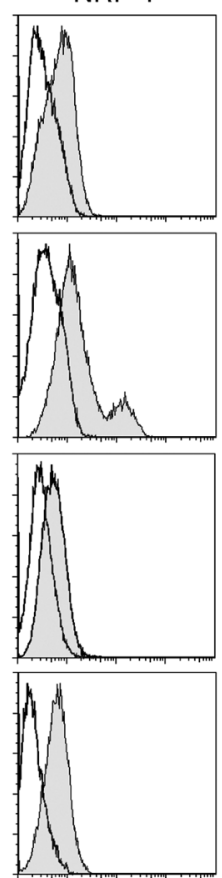

CD90
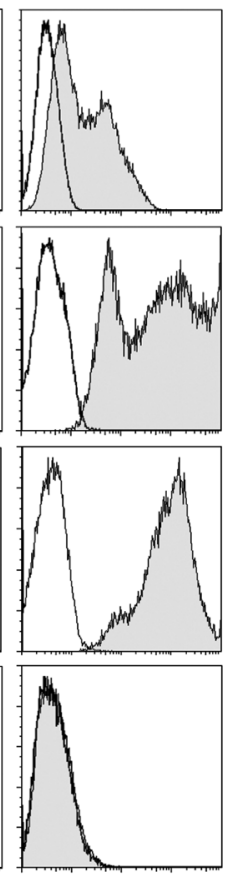

B

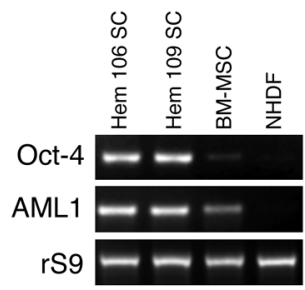

Figure 1

HemSCs express VEGF-Rs and mesenchymal cell marker CD90. (A) Flow cytometric analysis of HemSCs, BM-MSCs, NHDFs, and HDMECs. Each cell type was grown in the EBM-2/20\% FBS and assayed at passage 6. Gray histograms show cells labeled with FITC- or PE-conjugated antibodies. Black lines show isotype-matched control FITC- or PE-conjugated antibodies. Incubation with anti-CD31 and anti-VEGF-R2 was carried out following saponin permeabilization of HemSCs, BM-MSCs, and NHDFs. (B) RT-PCR analysis of Oct-4 and AML1 in 2 different HemSCs, Hem 106 and 109, with BM-MSCs and NHDFs shown for comparison. Ribosomal S9 (rS9) served as a control.

to hereafter as HemSCs, were expanded in an endothelial growth medium (endothelial basal medium 2 [EBM-2]/20\% FBS). Human umbilical cbEPCs (18), HDMECs, BM-mesenchymal stromal cells (BM-MSCs), and neonatal human dermal fibroblasts (NHDFs) were cultured under identical conditions and used for comparison. Flow cytometric analyses for endothelial and mesenchymal cell-surface markers showed that HemSCs were most similar to BM-MSCs and NHDFs (Figure 1A). HemSCs expressed CD90, a mesenchymal cell marker, and proteins that are displayed on ECs, such as VEGF-R1, VEGF-R2, neuropilin-1 (NRP-1), and CD146, but none of these are exclusive to the endothelial lineage. Hence, the cell-surface markers on HemSCs indicate a mesodermal or mesenchymal cell phenotype. Phase contrast images of HemSCs in culture are shown in Supplemental Figure 1 (supplemental material available online with this article; doi:10.1172/JCI33493DS1).

RT-PCR analyses showed that HemSCs expressed the embryonic SC transcription factor Oct-4 (Figure 1B), which is known to play a pivotal role in SC specification (19). HemSCs were also positive for AML1 (Figure 1B), a transcription factor required for definitive hematopoiesis (20) and expressed in hemogenic endothelium (21). Expression of Oct-4 and AML1 in HemSCs indicates that HemSCs are multipotent.

We next compared the growth parameters of HemSCs with normal mesenchymal cells and ECs. HemSCs grew to 3-4 times greater density than normal control endothelial and mesenchymal cells, which included HDMECs, cbEPCs, NHDFs, and BM-MSCs (Figure 2A). The proliferative response to serum, basic bFGF, and VEGF-A was determined after cells were serum and growth factor starved for 6 hours (Figure 2B). HemSCs and NHDFs proliferated in response to serum and $\mathrm{bFGF}$, although the response of HemSCs was stronger, as was the basal proliferation. HemSCs, BM-MSCs, and NHDFs did not show increased proliferation in response to VEGF-A, a well-studied endothelial growth and survival factor, while cbEPCs and HDMECs proliferated, as expected.

HemSCs display in vitro clonogenic and multilineage differentiation ability. To study cellular differentiation, we prepared clonal populations from the HemSCs from 3 different proliferating phase IH specimens. This was done by diluting a single-cell suspension of HemSC to 3 cells/ml and plating $100 \mu \mathrm{l}$ in each well of a 96-well plate; thereafter, wells were inspected to verify that only 1 colony was present in the well. Clonally expanded cells were necessary for multilineage assays of differentiation and later for in vivo studies to exclude the possibility that mixed populations of unipotential cells were responsible for the phenotypes of cells observed following the induction. HemSCs showed in vitro clonal expansion, with efficiency ranging from 6\%-30\% (6\% for Hem95, 23\% for Hem97, and $30 \%$ for Hem106). BM-MSCs and NHDFs failed to produce clonal populations under identical conditions. To induce cell differentiation toward specific lineages, culture conditions reported in the literature were employed. For endothelial differentiation, HemSCs were seeded at high density on fibronectin-coated plates in serum-free medium with VEGF-B $(22,23)$. Clonal HemSCs differentiated into ECs, shown by expression of the EC markers CD31 (also known as PECAM-1) and VE-cadherin (Figure 2C). Treatment with nerve growth factor and trans retinoic acid $(24,25)$ induced morphological changes, expression of the glial cell marker 

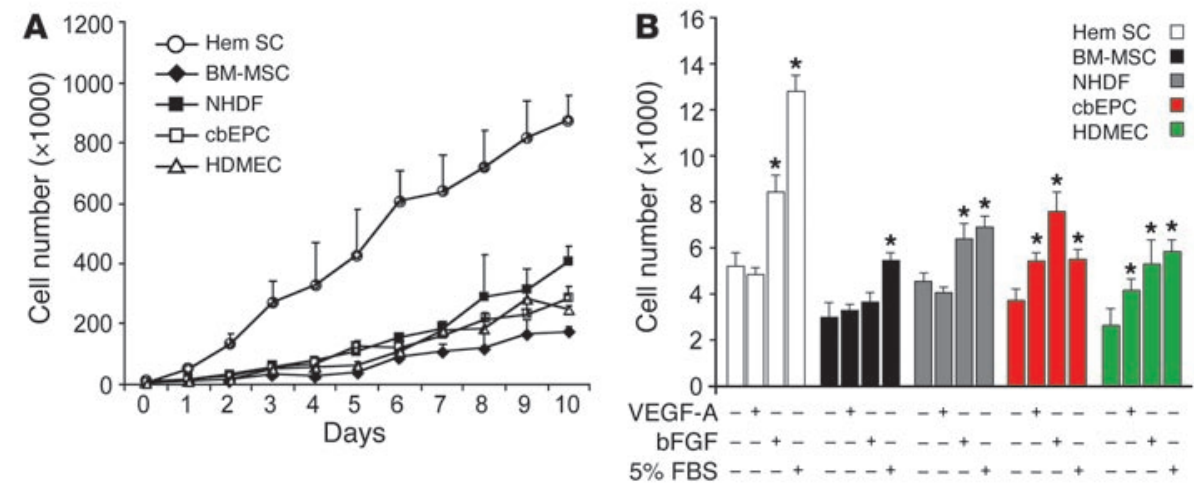

C

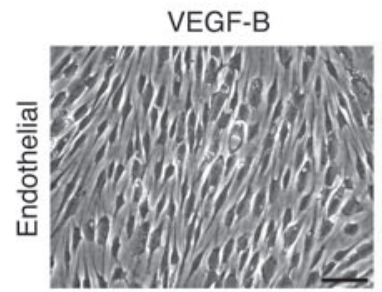

D
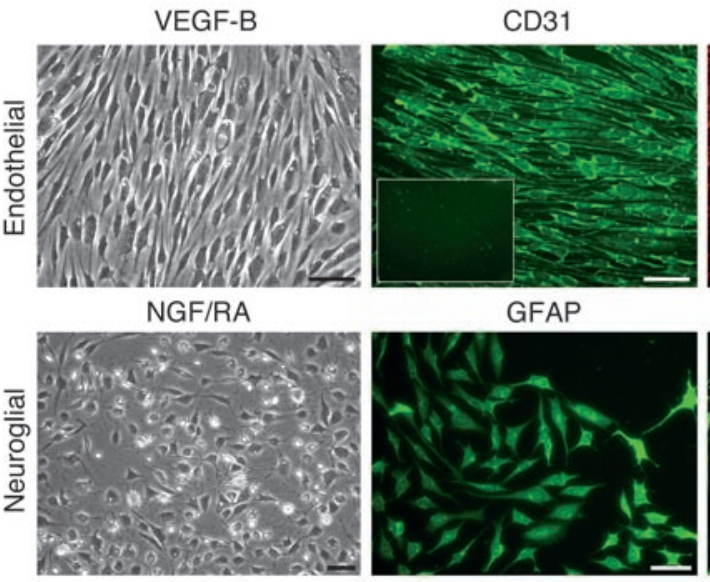

GFAP

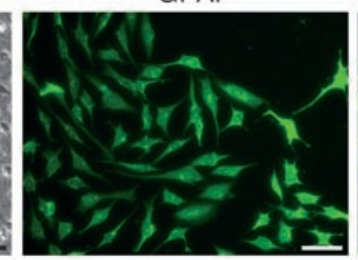

E

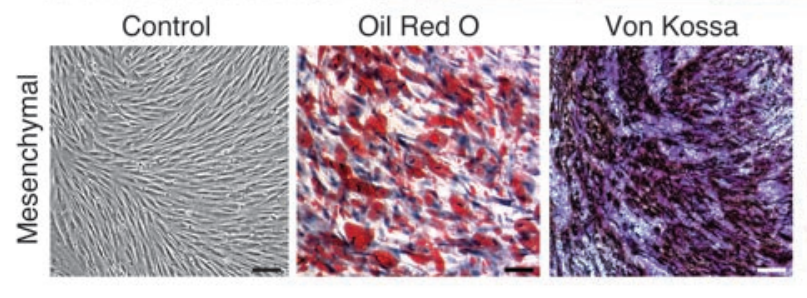

glial fibrillar acidic protein (GFAP), and the neuronal marker $\beta$-tubulin III (Figure 2D). Finally, mesenchymal differentiation into adipocytes, osteocytes, and chondrocytes was also seen (Figure 2E) when subjected to well-established protocols (26). BM-MSCs differentiated into fat, bone, and cartilage as expected, whereas NHDFs failed to differentiate into any other cell type in the same inductive medium (data not shown). Interestingly, HemSCderived ECs exhibited an intracellular localization of CD31 and VE-cadherin (Figure 2C), reminiscent of an earlier study of hemangioma-derived and fetal ECs (10). These findings indicate that other signals or factors may be required to fully differentiate the HemSCs into mature ECs with the expected localization of adhesion molecules at the cell surface. Some IH blood vessels coexpress myeloid markers (27), further illustrating the unique phenotype of $\mathrm{IH}$ endothelium. As hematopoietic cells and ECs arise from a common progenitor during development (28), we tested to determine whether HemSCs would produce hematopoietic colonies in vitro. Incubation of clonally expanded HemSCs in MethoCult medium did not yield hematopoietic colonies (data not shown).

HemSCs form hemangioma-like Glut-1 $1^{+}$blood vessels in immunodeficient mice. We tested to determine whether HemSCs would form hemangiomas in immunodeficient $\mathrm{nu} / \mathrm{nu}$ mice. Clonally expanded
VE-cadherin

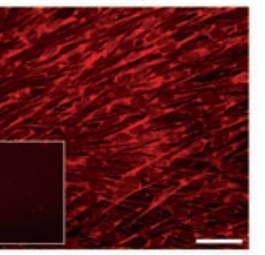

$\beta$-tubulin III

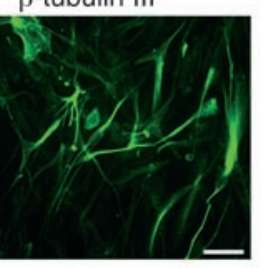

Coll type II

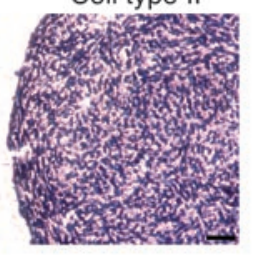

\section{Figure 2}

In vitro growth and multilineage differentiation of HemSCs. (A) Proliferation of HemSCs in EBM-2/20\% FBS over 10 days compared with normal endothelial and mesenchymal cells. (B) Proliferation in response to VEGF-A, bFGF, or $5 \%$ FBS for 24 hours in serum-free, growth factor-free EBM medium. ${ }^{*} P<0.05$ compared with cells in serum-free, growth factor-free medium. (C-E) Clonal HemSCs differentiated into endothelial (C), neuroglial (D), and mesenchymal (E) cells. Scale bars: $50 \mu \mathrm{m}$. Insets in C show CD31 and VE-cadherin immunostaining of cells induced in the absence of VEGF-B. All experiments were carried out with cells at passages 6-9.

HemSCs or normal control cells were resuspended in Matrigel, a solubilized basement membrane preparation that has been shown to support angiogenesis and vasculogenesis $(18,29,30)$. Cells were injected s.c. into 6 -week-old mice $\left(1.5 \times 10^{6}\right.$ cells/200 $\left.\mu \mathrm{l} \mathrm{Matrigel/animal}\right)$. $\mathrm{H} \& \mathrm{E}$ staining of sections showed numerous rbc-filled vascular channels 7 and 14 days after implantation. After 28 and 56 days in vivo, vessels were still present, but large lipid-filled cells became prominent (Figure 3A). Similar results were obtained with nonclonally expanded HemSCs from 4 different hemangioma specimens (data not shown). Blood vessels were not detected when HemECs (3), HemEPCs (13), hemangioma-derived mesenchymal SCs (HemMSCs) (31), BM-MSCs, cbEPCs (18), or NHDFs were implanted as single-cell types into mice under identical conditions (Supplemental Figure 2).

To verify that the newly formed blood vessels were composed of human ECs, we performed indirect immunofluorescence using an antibody specific for human CD31. Immunostained sections were examined by confocal microscopy. Human CD31 was detected in blood vessels formed from clonal HemSCs, indicating endothelial differentiation and vasculogenesis (Figure 3B). The specificity of the anti-human CD31 was confirmed by lack of staining in mouse tissues (data not shown) and has been demonstrated in other studies $(18,32)$. We next determined whether the human CD31-positive vessels expressed the GLUT-1-positive immunophenotype, which has been shown to be a specific signature of IH (33). The blood vessels formed from HemSCs in Matrigel were found to coexpress CD31 and GLUT-1 (Figure 3B). High magnification images with DAPIstained nuclei are shown in Figure 3B to highlight the coexpression of CD31 and GLUT-1. A second marker of the hemangioma phenotype, merosin (34), was also found expressed in the hemangioma lesions formed in mice from HemSCs (Supplemental Figure 3).

To further examine human CD31 expression in the Matrigel model, cells were reisolated from the explants by digestion with a collagenase/dispase solution. Flow cytometry was performed with- 
A
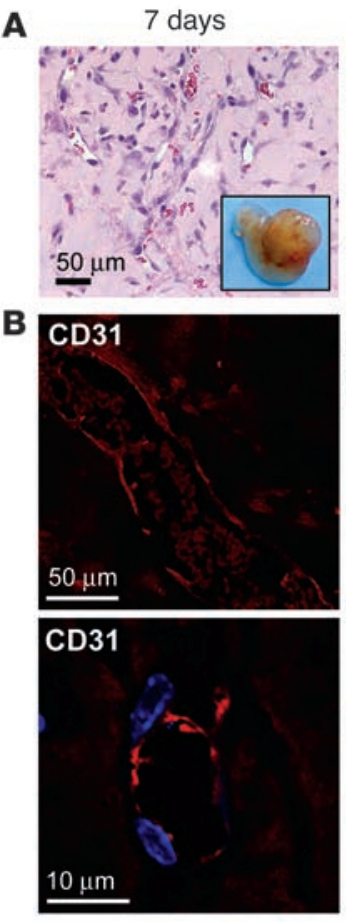

C

Hem SC

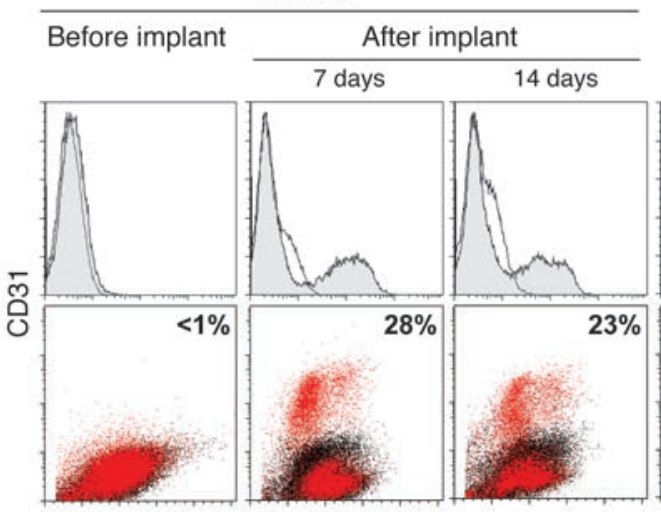

14 days
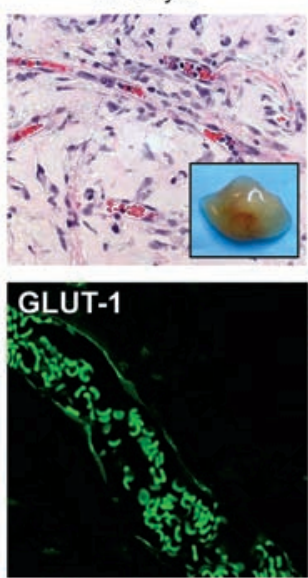

\section{GLUT-1}

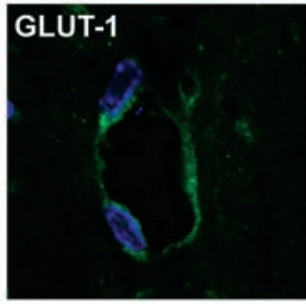

-

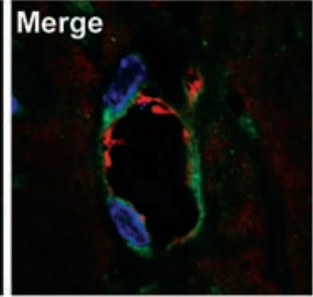

28 days

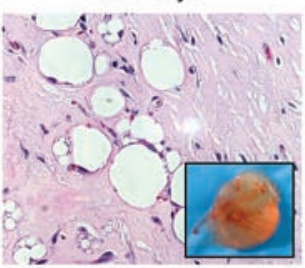

\section{Merge}

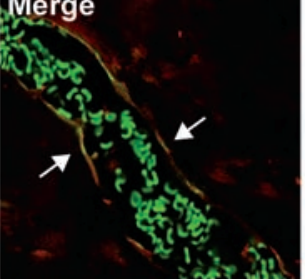

Merge

Mouse EC

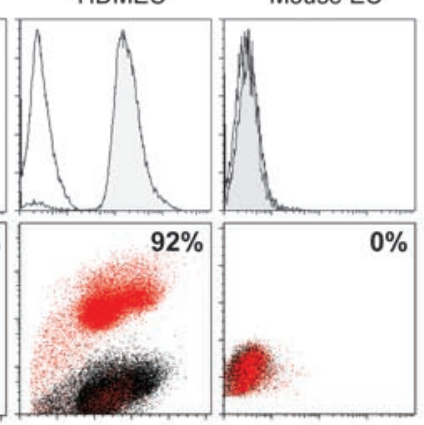

56 days
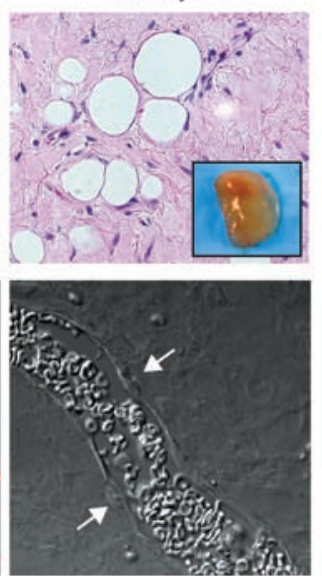

D
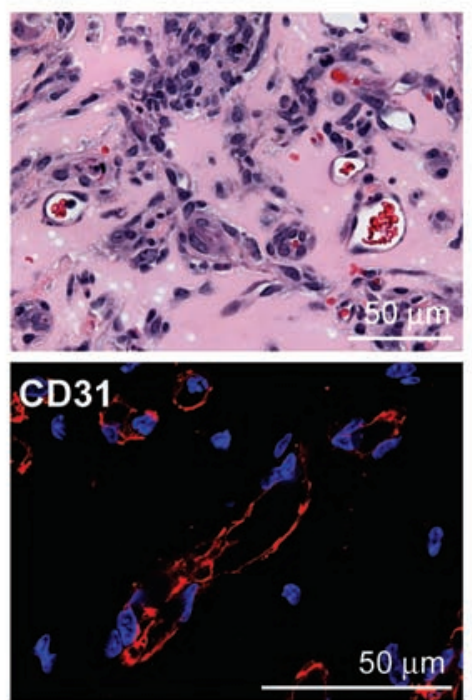

\section{Figure 3}

HemSCs form CD31+GLUT-1+ blood vessels in vivo. (A) Clonal HemSCs were suspended in Matrigel and injected s.c. into nude mice. H\&E sections of explants at $7,14,28$, and 56 days with insets showing explants at the corresponding time points. Scale bar: $50 \mu \mathrm{m}$. Passage 9 clonal HemSCs were used. (B) Immunofluorescent staining of day 7 explants. Human CD31 (red) is shown on the left, followed by GLUT-1 (green), a merged image, and a phase contrast image. In the merged image, arrows point to cells along the lumen of the blood vessel that are double-labeled for human CD31 and GLUT-1. rbc in the lumen are also positive for GLUT-1 (green). In the phase image, arrows point to endothelial nuclei. The bottom row shows higher magnification images. Scale bars: $50 \mu \mathrm{m}$ (top row); $10 \mu \mathrm{m}$ (bottom row). (C) Flow cytometry of cells reisolated from Matrigel explants after 7 and 14 days in vivo. Cells were labeled with anti-human CD31 without permeabilization to detect surface-localized CD31. HemSCs before implantation were negative for CD31 (left panels). HDMECs and mouse ECs are shown as positive and negative control cells. (D) Retrieved CD31+ cells formed blood vessels in secondary recipient mice. Matrigel implants were removed after 14 days, sectioned, stained with H\&E (top panel), and immunostained with anti-human CD31 (bottom panel). Vessel density was $78 \pm 35$ rbc-filled lumens $/ \mathrm{mm}^{2}$. Scale bars: $50 \mu \mathrm{M}$.

out permeabilization of the cells with anti-human CD31 (Figure $3 \mathrm{C})$. After 7 and 14 days in vivo, $23 \%-28 \%$ of the retrieved cells were found to express human CD31 on the surface. This is consistent with functional endothelial differentiation, demonstrated by blood vessel formation (Figure 3, A and B), and provides additional evidence for the differentiation of HemSCs into ECs.

To further test the stem/progenitor nature and vasculogenic potential of these cells, the CD31-positive cells retrieved from the Matrigel were expanded in vitro in EBM-2/20\% FBS, verified as human cells by immunostaining for a human nuclear antigen (Supplemental Figure 4), and injected into secondary recipient nude mice. After 14 days, Matrigel explants were removed, sectioned, and analyzed by H\&E to quantify rbc-filled lumens and immunostain for human CD31 (Figure 3D). Vessel density was $78 \pm 35$ vessels $/ \mathrm{mm}^{2}$, which was greater than that observed in primary recipients. The ability to retrieve HemSCs that had undergone 

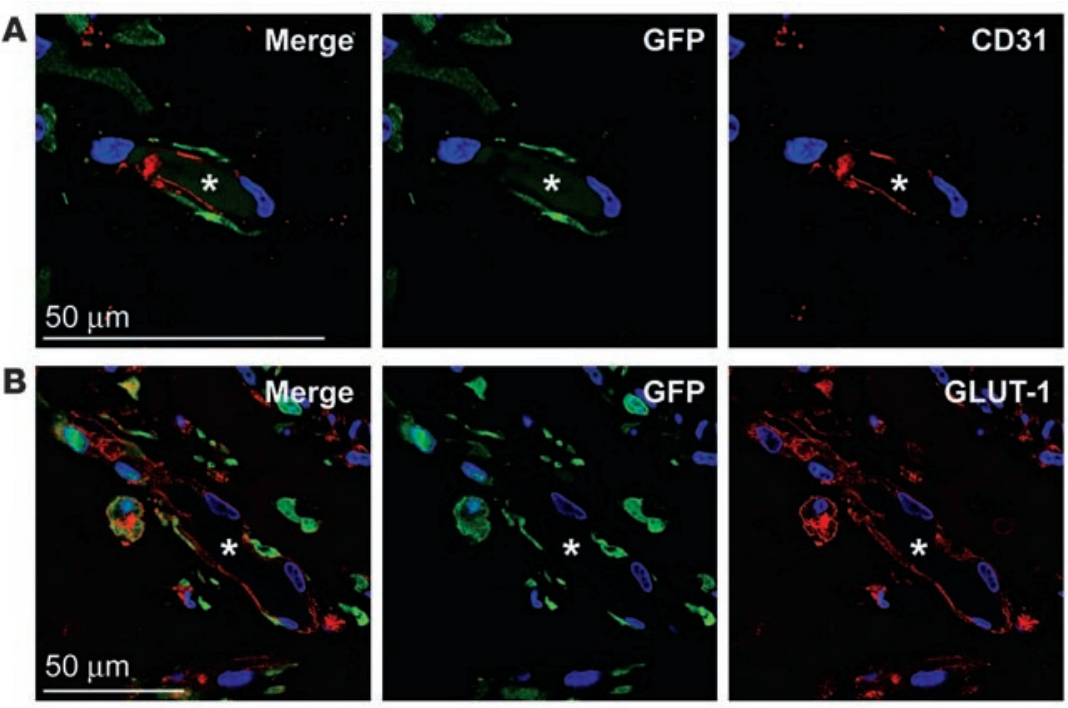

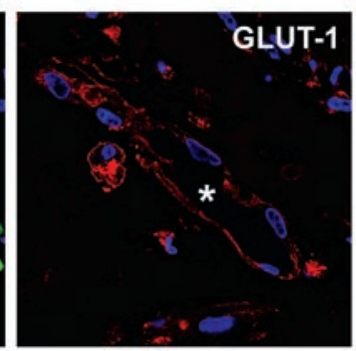

\section{Figure 4}

GFP-labeled HemSCs form hemangioma blood vessels in vivo. (A) TurboGFP-expressing HemSCs were injected in Matrigel into mice, explanted at day 14 , and localized by staining for TurboGFP (green), CD31 (red), and DAPI (blue). (B) Sections were also stained for TurboGFP (green), GLUT-1 (red), and DAPI (blue). Asterisks mark the lumen of the blood vessels in panels A and B. Scale bars: $50 \mu \mathrm{M}$.

the anti-GFP antibody are shown in Supplemental Figure 6. These data combined with that shown in Figure $3 \mathrm{~B}$ demonstrate that vessels formed when HemSCs were implanted into nude mice were composed of human ECs; the vessels were not the result of infiltration by murine ECs.

In vivo differentiation of HemSCs to adipocytes. Our next objective was to determine whether HemSCs recapitulated the involuting and involuted endothelial differentiation in vivo and to obtain hemangioma-like blood vessel formation in secondary recipients demonstrates the robust blood vessel-forming potential of the HemSCs.

GFP-labeled HemSCs form blood vessels. To track the cellular fate of the HemSCs in our in vivo model, HemSCs were infected with a lentiviral construct encoding turbo GFP. GFP-positive cells were shown by flow cytometry to be greater than $90 \%$ positive for GFP expression (Supplemental Figure 5). The cells were further expanded in vitro for 1 passage and implanted into nude mice under conditions identical to those used in the previous experiments (Figure 3). Matrigel implants were harvested at 2 weeks and analyzed for GFP, human CD31, and GLUT-1. GFP and CD31 were colocalized in lumenal structures indicative of blood vessels (Figure 4A). GFP-positive cells lining vessels were also positive for the hemangioma vessel marker GLUT-1 (Figure 4B). Controls to rule on nonspecific staining with phases of IH. After 28 days in vivo, HemSC-formed lesions had diminished microvessel density (MVD), and adipocytes emerged between 28 and 56 days (Figure 5A and Figure 3A). Morphometric quantification showed 25 vessels $/ \mathrm{mm}^{2}$ at days 7,14 , and 28 , which decreased to 15 vessels $/ \mathrm{mm}^{2}$ at 56 days (Figure $5 \mathrm{~A}$ ), consistent with the reduced number of vascular channels seen during the evolution of IH. It has been reported that Matrigel supplemented with bFGF induces adipogenesis in vivo (35-37). However, no exogenous bFGF is included in our assay. We tested to determine whether the adipocytes in the Matrigel were host derived (murine) or human and had differentiated from the clonal HemSCs. To do this, GFP-HemSCs were implanted, as described in Figure 4, and removed after 2 months in vivo for analysis of GFP and perilipin A (38). Perilipin A encircled the lipid storage droplets in adipocytes, as expected (Figure 5B). GFP and perilipin A colocalization was
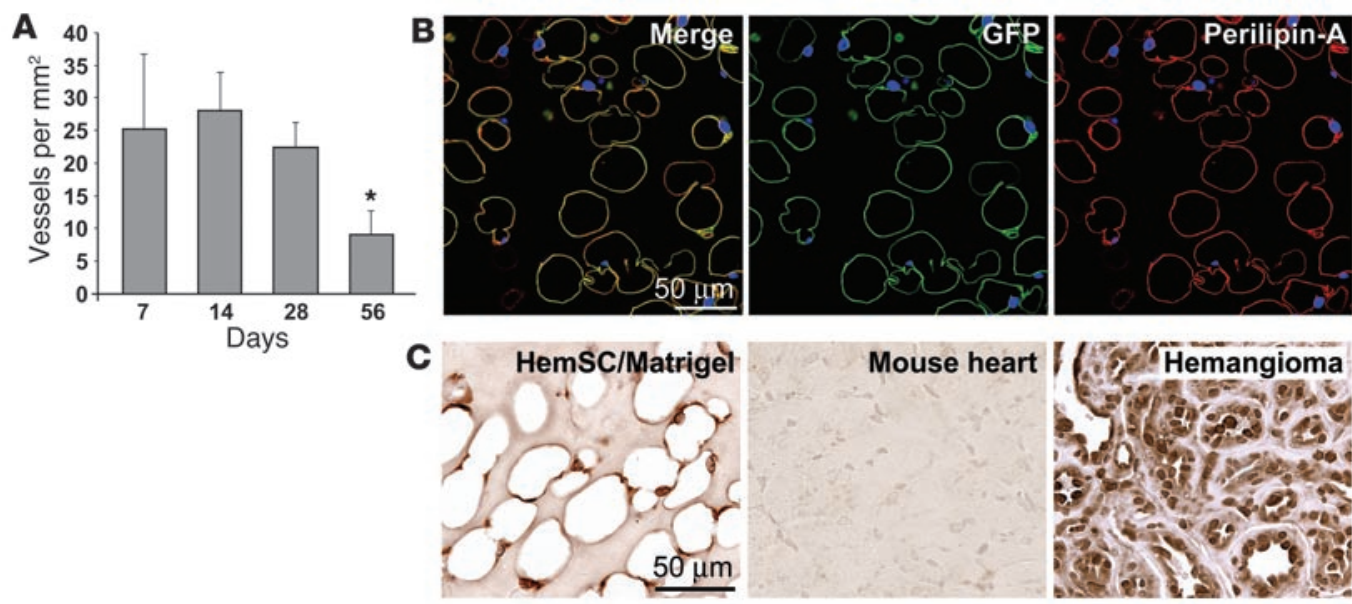

Mouse heart I / 5 Sed Hemangioma

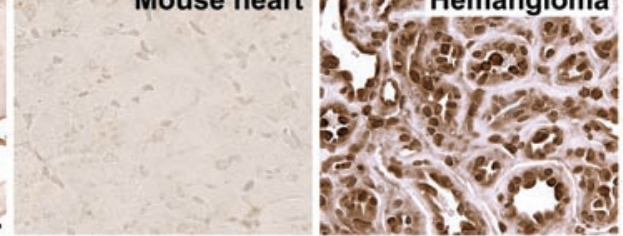

\section{Figure 5}

HemSCs form adipocytes in vivo. (A) Clonal HemSCs were suspended in Matrigel and injected s.c. into nude mice for $7,14,28$, and 56 days, as shown in Figure 3A. Blood vessels, identified in H\&E sections as luminal structures containing rbc, were quantified at the 4 time points. ${ }^{*} P<0.05$ compared with other time points. (B) GFP-labeled HemSCs were implanted as described in Figure 4 and analyzed after 56 days for expression of GFP and the adipocyte marker perilipin A. Right panel shows the merged image, middle panel shows GFP (green), and right panel shows perilipin A (red); all panels were stained with DAPI. (C) Sections from the 28-day time point were immunostained with an anti-human nuclear antigen antibody using an HRP-conjugated secondary antibody and a diaminobenzidine substrate. Mouse heart tissue sections showed no immunostaining while nuclei in human hemangioma tissue were uniformly positive, confirming the specificity of anti-human nuclear antigen. Scale bars: $50 \mu \mathrm{M}$. 
seen in the scant cytoplasm of the lipid-filled adipocytes (Figure $5 \mathrm{~B})$. Controls to rule out nonspecific staining with the anti-GFP and anti-perilipin A antibodies are shown in Supplemental Figure 6. As an alternative approach to follow HemSC adipogenesis in vivo, sections were immunostained with an anti-human nuclear antigen antibody (Figure 5C). The human nuclear antigen was detected in the nuclei of adipocytes in the HemSC Matrigel implants. Mouse heart tissue sections served as a negative control, and human hemangioma tissue sections served as a positive control (Figure 5C). Some adipocytes negative for the human nuclear antigen were evident, suggesting that recruitment of murine cells into the Matrigel microenvironment may have occurred. These results with tagged, GFP-labeled HemSCs and the anti-human nuclear antigen antibody indicate that HemSCs form adipocytes at time points subsequent to vessel formation. The signals that determine whether and when a HemSC becomes an EC or an adipocyte are unknown.

\section{Discussion}

For what we believe is the first time, we have shown that CD133selected HemSCs recapitulate human IH in a murine in vivo model. Clonal HemSCs produced human GLUT-1-positive microvessels at 7-14 days; after 28 days in vivo, human adipocytes were also detected. HemSCs that had differentiated into endothelium in vivo, as determined by cell-surface CD31 expression, could be retrieved and implanted into secondary recipients, where the cells formed blood vessels once more. This indicates the robust vasculogenic potential of these hemangioma-derived cells. GFP-labeled HemSCs were shown to form vessels and adipocytes at 14- and 56-day time points, respectively, confirming that the vessels and adipocytes were not host derived. Using in vitro cellular activity assays, we showed that HemSCs are highly proliferative and able to differentiate into multiple cell types, including adipocytes. These results demonstrate that HemSCs are the cellular precursors of $\mathrm{IH}$.

CD133 has been used to identify tumor-initiating cells from medulloblastomas, glioblastomas, and prostate and colon carcinomas $(16,17,39-42)$. Xenografting CD $133^{+}$tumor cells in immune-compromised mice has also been shown to generate a primary tumor $(16,17)$. In a similar fashion, we demonstrated that the CD133-selected cells from IH formed GLUT-1-positive blood vessels and, at later time points, differentiated into adipocytes, the prominent cellular compartments of proliferating and involuting phase hemangioma, respectively. For the secondary transplantation experiment (Figure 3, C and D), we reimplanted human CD31-selected cells because (a) these cells had shown vasculogenic activity in the primary implant and (b) the level of CD133 expression in the CD31-positive and CD31-negative populations recovered from the primary implant was very low, which would have made reselecting CD133-positive cells difficult. The human CD31-selected cells showed a robust degree of blood vessel formation $\left(78\right.$ vessels $/ \mathrm{mm}^{2}$ ) upon secondary transplantation, a finding that supports the vasculogenic potential of these cells.

To date, there are no animal models that truly reflect the human hemangioma phenotype and life cycle. Although murine hemangioendothelioma cells have been studied (43), these do not phenocopy IH or provide a hemangioma model because the tumors do not regress and are fatal within 3 months. In addition, polyoma middle $\mathrm{T}$ antigen-transformed cells used in animal models result in "cavernous hemangioma" lesions, more akin to malignant vascular tumors, which are also fatal within 3-10 months (44). Hem- angioma tissue fragments xenografted into nude mice have been shown to undergo an initial period of necrosis followed by approximately 2-fold enlargement and then subsequent regression (45), which may provide a model for testing new therapies for IH. Our in vivo model is unique in that we are able to produce IH lesions with a small number of purified primary cells suspended in Matrigel but without exogenous growth factors or supplements. These findings implicate a preset genetic or epigenetic program in the HemSCs that directs the cells to recapitulate the IH tumor, including endothelial and adipocytic differentiation. An important future experiment will be to implant hemangioma-derived $\mathrm{CD} 133^{+}$cells that have not undergone in vitro expansion to determine whether HemSCs lose or gain hemangioma-forming potential during the in vitro culture. It is still not understood what triggers the adipogenesis that characterizes the involuting and involuted phases of IH. It is likely that changes in the microenvironment, such as levels of circulating EPCs, immune cells, and growth factors, initiate the regression of the tumor.

It is well established that hemangioma endothelium expresses GLUT-1, merosin, Lewis Y antigen (CD174), and Fc $\gamma$ receptor II (CD32) in all 3 phases of the life cycle (34). These markers are also expressed by placental microvessels. This immunohistochemical similarity suggested the hypothesis that $\mathrm{IH}$ is formed from embolic placental ECs (34). Microarray analyses have supported this link between placenta and hemangioma (46). It is also possible that the progenitor cells reside in fetal and neonatal tissues or come from the BM and express a transcriptional program similar to that of placenta. To date, placenta-derived cells with in vitro clonogenic and differentiation abilities similar to HemSCs have not been described.

In conclusion, we have identified the hemangioma-initiating cell. We show that a single multipotential cell (HemSC) produces GLUT-1-positive microvessels and that this lesion undergoes an involutive process by differentiation into adipocytes. This could be a result of a somatic mutation within a stem or progenitor cell and subsequent expansion/differentiation. It is also plausible that a normal SC in the context of the postnatal infantile microenvironment becomes disrupted or delayed in its differentiative program, resulting in an accumulation of early progenitor cells. Such a clonal population could then undergo ill-timed or abnormal differentiation, which subsequently resolves. Understanding the cellular origin of IH provides an avenue for deciphering the mechanisms of EC differentiation and delineating targets for adjuvant therapy. Our in vitro and in vivo models of IH offer powerful tools to evaluate potential therapeutic modalities and insight into the biology of de novo formation of blood vessels.

\section{Methods}

Cell isolation and culture. Specimens of proliferating $\mathrm{IH}$ were obtained from the Department of Plastic Surgery at Children's Hospital Boston under a human subject protocol that was approved by the Committee on Clinical Investigation. The clinical diagnosis was confirmed in the Department of Pathology at Children's Hospital Boston. Informed consent was provided for the specimens, according to the Declaration of Helsinki. Singlecell suspensions were prepared from the proliferating phase specimens as described previously (13). HemSCs were selected using anti-CD133-coated magnetic beads (Miltenyi Biotec). CD133-selected cells were cultured on fibronectin-coated $\left(1 \mu \mathrm{g} / \mathrm{cm}^{2}\right)$ plates with EBM-2 (CC-3156; Cambrex). EBM-2 was supplemented with $20 \%$ FBS, endothelial growth media-2 SingleQuot (CC-4176; Cambrex), and $1 \times$ PSF (100 U/ml penicillin, 
$100 \mu \mathrm{g} / \mathrm{ml}$ streptomycin, $0.25 \mu \mathrm{g} / \mathrm{ml}$ amphotericin; Invitrogen). Hereafter this supplemented medium is called EBM-2/20\% FBS.

Neonatal foreskin and umbilical cord blood were obtained with institutional research board approval from Brigham and Women's Hospital, Boston, Massachusetts, USA. HDMECs were isolated as described (13). Human umbilical cbEPCs were isolated with anti-CD133-coated magnetic beads (Miltenyi Biotec). In addition, BM-MSCs and NHDFs were obtained commercially (Cambrex) and cultured on fibronectin-coated plates in EBM-2/20\% FBS under conditions identical to those used for the HemSCs. For in vivo experiments and in vitro differentiation studies, individually expanded clones of HemSCs were prepared by a limiting dilution technique in which cells were diluted in EBM-2/20\% FBS media to 3 cells $/ \mathrm{ml}$ and dispensed at $100 \mu \mathrm{l}$ per well in 96 -well dishes. After plating and until colonies were harvested, wells were inspected to insure that only 1 colony per well was present.

To obtain GFP-expressing cells, $1.6 \times 10^{4} \mathrm{HemSCs}$ were plated into wells of a 96-well plate. After 24 hours, fresh medium containing $8 \mu \mathrm{g} / \mathrm{ml}$ hexadimethrine bromide (Sigma-Aldrich) was added and cells were then infected with $10 \mu$ lentiviral-based particles containing TurboGFP encoding vector (Mission TurboGFP Control Vector; Sigma-Aldrich). To obtain stable GFP expression, cells were maintained in $2 \mu \mathrm{g} / \mathrm{ml}$ puromycin medium (Sigma-Aldrich).

Flow cytometry. Cells were labeled with FITC-conjugated mouse antihuman CD31 (Ancell), PE-conjugated mouse anti-human CD34 (Miltenyi Biotec), FITC-mouse anti-human CD146 (Millipore), PE-mouse anti-human CD90 (Millipore), and PE-mouse anti-human neuropilin-1 (NRP-1; Miltenyi Biotec). Cells were also incubated with rabbit anti-human VEGF-R1 (Santa Cruz Biotechnology Inc.) and mouse anti-human VEGFR2 (Clone KDR1; Sigma-Aldrich) antibody followed by FITC-conjugated secondary antibody (Vector Laboratories). VEGF-R1, VEGF-R2, and CD34 labeling were carried out after permeabilization of the cells with $0.5 \%$ saponin (Sigma-Aldrich). Flow cytometry was performed on a BD FACScan. Data were analyzed using FlowJo software.

RT-PCR. Total RNA was extracted from cultured cells using RNeasy Mini Kit (QIAGEN). Purity of the RNA samples was assessed by determining the optical density at 260:280 nm. Three micrograms of total RNA was used for cDNA synthesis using iScript cDNA Synthesis Kit (Bio-Rad). Primers for Oct-4 (47), AML1 (48), and ribosomal S9 (13) were used. All reactions were performed for 30 cycles with the following temperature profiles: $95^{\circ} \mathrm{C}$ for 3 minutes (initiation; $30 \mathrm{~s} /$ cycle thereafter); $55^{\circ} \mathrm{C}$ for 45 seconds (annealing); and $72^{\circ} \mathrm{C}$ for 45 seconds (extension). Size of the PCR products was confirmed by running $10 \mu \mathrm{l}$ of the reaction product on $3 \%$ agarose gel.

In vivo murine model of $I H$. All experiments were carried out with $1.5 \times 10^{6}$ cells per animal. Cells were trypsinized, counted, and resuspended in phenol red-free Matrigel (BD Biosciences). The cell/Matrigel mixture (200 $\mu \mathrm{l} /$ animal) was injected s.c. into the backs of 6 -week-old male athymic $\mathrm{nu} / \mathrm{nu}$ mice ( $n=4$ /group; Massachusetts General Hospital, Boston, Massachusetts, USA). The animals were monitored for weight gain and Matrigel plug size at 7-day intervals throughout the period of the study. Animals were sacrificed and Matrigel plugs harvested at 7, 14, 28, and 56 days. The Matrigel plugs were then either fixed in $10 \%$ neutral buffered formalin for immunohistochemistry (described below) or used immediately for cellretrieval experiments. Cell retrieval was performed by incubating the Matrigel explants in DMEM medium supplemented with 2\% FBS and 4x PSF for 1 hour. The explants were then digested with $0.2 \%$ collagenase A and 2.5 $\mathrm{U} / \mathrm{ml}$ dispase (BD Biosciences). rbc were lysed by incubating the cell suspension with chilled $\mathrm{NH}_{4} \mathrm{Cl}$ (StemCell Technologies). The cells were labeled with FITC-mouse anti-human CD31 antibody as described above. Mouse dermal ECs (kindly provided by Michael Klagsbrun, Children's Hospital Boston) were used to determine the specificity of the CD31 antibody.
Immunohistochemistry. Immunohistochemistry was performed on formalin-fixed/paraffin-embedded Matrigel plugs. The sections were deparaffinized in xylene and hydrated through sequential ethanol gradient. $H \& E$ images were captured with Axiophot II fluorescence microscope (Zeiss) equipped with AxioCam MRc5 (Zeiss). Antigen retrieval was carried out by heating the sections in citrate-EDTA buffer $(10 \mathrm{mM}$ citric acid, 2 mM EDTA, $0.05 \%$ Tween-20, pH 6.2) or Tris-EDTA buffer (10 $\mathrm{mM}$ Trizma-base, $1 \mathrm{mM}$ EDTA, $0.05 \%$ Tween-20, $\mathrm{pH} 9)$. The sections were blocked for 30 minutes in 5\% serum and incubated with rabbit anti-human GLUT-1 antibody (1:50; A3536, Dako) or mouse antihuman GLUT-1 antibody (1:25; ab40084, Abcam), followed by FITC or Texas red-labeled secondary antibody (1:200; Vector Laboratories). The sections were washed and blocked again for 30 minutes. Humanspecific CD31 monoclonal antibody (1:50; M0823 clone JC/70A, Dako) followed by Texas red anti-mouse secondary antibody (1:200; Vector Laboratories) was used for detection of human-specific microvessels. Sections were also stained for merosin (1:50; MAB1922 clone 5H2, Millipore), human-specific nuclear antigen (HuNuc, 1:25; MAB 1281 clone 235.1, Millipore), and rabbit anti-human perilipin A (perA, 1:100; P1998, Sigma-Aldrich) or goat anti-human perilipin A (1:200; ab61682, Abcam). The human nuclear antigen staining was performed using an HRP-conjugated secondary antibody followed by incubation with diaminobenzidine solution (Vector Laboratories). GFP expression was detected with anti-TurboGFP antibody (1:2000; AB511, Evrogen). The sections were counterstained with DAPI (Vector Laboratories). All fluorescent images were taken with a Leica TCS SP2 Acousto-Optical Beam Splitter confocal system equipped with DMIRE2 inverted microscope (diode $405 \mathrm{~nm}$, argon $488 \mathrm{~nm}$, HeNe $594 \mathrm{~nm}$; Leica Microsystems).

$M V D$. For the assessment of MVD, 4 fields from mid-Matrigel H\&E sections of each of the 4 animals in the group were used. Microvessels were quantified by counting luminal structures containing rbc. MVD was calculated as vessels $/ \mathrm{mm}^{2}$.

Assays for in vitro cellular activity. HemSCs were subjected to cellular activity assays that measured cell growth, proliferation in response to growth factors, and differentiation. Growth parameters were assessed by seeding the cells at 5000 cells $/ \mathrm{cm}^{2}$ on fibronectin-coated 48-well plates (4000 cells; growth surface, $0.8 \mathrm{~cm}^{2}$ ) and culturing in EBM-2/20\% FBS. The cells were counted every day using a hemocytometer, and the medium was changed every 2 days. For proliferation experiments, cells were plated as described above. Following attachment ( 24 hours), plating efficiency was determined, medium was removed, and cells were serum starved for 6 hours. Proliferation was assessed after 24 hours in cells exposed to serum-free medium, $10 \mathrm{ng} / \mathrm{ml}$ VEGF-A (R\&D Systems), $10 \mathrm{ng} / \mathrm{ml}$ VEGF-B (R\&D Systems), $5 \mathrm{ng} / \mathrm{ml}$ bFGF (Roche Applied Science), or 5\% FBS.

To study multilineage differentiation, cells were treated with induction media for 14 days. Mesenchymal differentiation of the cells was carried out with adipogenic media (DMEM/10\% FBS, $5 \mu \mathrm{g} / \mathrm{ml}$ insulin, $1 \mu \mathrm{M}$ dexamethasone, $0.5 \mathrm{mM}$ isobutylmethylxanthine, $60 \mu \mathrm{M}$ indomethacin, and $1 \times$ PSF), osteogenic media (DMEM/10\% FBS, $1 \mu \mathrm{M}$ dexamethasone, 10 $\mathrm{mM} \beta$-glycerophosphate, $60 \mu \mathrm{M}$ ascorbic acid-2-phosphate, and $1 \times \mathrm{PSF}$ ), and chondrogenic media (DMEM high glucose, $1 \times$ insulin-transferrinselenium, 1:5000 linoleic acid-albumin, $1 \mu \mathrm{M}$ dexamethasone, $100 \mu \mathrm{M}$ ascorbic acid-2-phosphate, $10 \mathrm{ng} / \mathrm{ml} \mathrm{TGF-} \beta 1$, and $1 \times$ PSF). EC differentiation was carried out in serum-free medium containing VEGF-B (EBM-2, $1 \times$ insulin-transferrin-selenium, 1:5000 linoleic acid-albumin, $1 \mu \mathrm{M}$ dexamethasone, $60 \mu \mathrm{M}$ ascorbic acid-2-phosphate, $10 \mathrm{ng} / \mathrm{ml}$ VEGF-B). For neurogenic differentiation, cells were exposed to initiating medium (DMEM/10\% FBS, $1 \mathrm{mM} \beta$-mercaptoethanol) for 24 hours. Neurogenic induction medium (DMEM, $0.1 \mu \mathrm{M}$ dexamethasone, $0.25 \mu \mathrm{g} / \mathrm{ml}$ insulin, $5 \mu \mathrm{M}$ cAMP, $0.5 \mathrm{mM}$ isobutylmethylxanthine, $5 \mu \mathrm{M}$ trans-retinoic acid, 
$0.25 \mathrm{mM}$ L-glutamine, and $25 \mathrm{ng} / \mathrm{ml}$ nerve growth factor) was then used for 14 days. Hematopoietic activity was assessed in MethoCult medium (StemCell Technologies) at $1 \times 10^{3}, 1 \times 10^{4}, 1 \times 10^{5}$, and $1 \times 10^{6}$ cells per $35-\mathrm{mm}$ dish. Mesenchymal differentiation was assessed by oil red $\mathrm{O}$ (lipid droplets), alkaline phosphatase induction (substrate-based activity) and Von Kossa (mineralization), and collagen type 2 staining. Immunofluorescent analysis of cells was carried out using goat anti-human CD31 (Santa Cruz Biotechnology Inc.), mouse anti-human VE-cadherin (Immunotech), mouse anti-human tubulin $\beta$-tubilin III (Millipore), and goat anti-human GFAP (Dako) antibodies. FITC- and PE-conjugated secondary antibodies (Vector Laboratories) were used for antigen detection. Images were taken with a Nikon Eclipse TE300 (Nikon) using SPOT Advanced 3.5.9 software (Diagnostic Instruments Inc.) and a $\times 20 / 0.45$ objective lens.

Statistics. The data were expressed as means \pm SEM and analyzed by ANOVA followed by Student's two-tailed $t$ test where appropriate. Differences were considered significant at $P<0.05$.

\section{Acknowledgments}

This work was supported by an NIH grant (P01 AR048564 to J. Bischoff). We thank Jill Wylie-Sears for her technical assistance, Elke Pravda for confocal microscopic imaging, and Kristin Johnson for the preparation of the figures.

Received for publication August 2, 2007, and accepted in revised form April 23, 2008.

Address correspondence to: Joyce Bischoff, Vascular Biology Program and Department of Surgery, Children's Hospital Boston, Harvard Medical School, Boston, Massachusetts 02115, USA. Phone: (617) 919-2192; Fax: (617) 730-0231; E-mail: joyce. bischoff@childrens.harvard.edu.

\section{Zia A. Khan and Elisa Boscolo are co-first authors.}

1. Mulliken, J.B., Fishman, S.J., and Burrows, P.E. 2000. Vascular anomalies. Curr. Probl. Surg. 37:517-584.

2. Bischoff, J. 2002. Monoclonal expansion of endothelial cells in hemangioma: an intrinsic defect with extrinsic consequences? Trends Cardiovasc. Med. 12:220-224.

3. Boye, E., et al. 2001. Clonality and altered behavior of endothelial cells from hemangiomas. J. Clin. Invest. 107:745-752.

4. Bielenberg, D.R., et al. 1999. Progressive growth of infantile cutaneous hemangiomas is directly correlated with hyperplasia and angiogenesis of adjacent epidermis and inversely correlated with expression of the endogenous angiogenesis inhibitor, IFNbeta. Int. J. Oncol. 14:401-408.

5. Berard, M., et al. 1997. Vascular endothelial growth factor confers a growth advantage in vitro and in vivo to stromal cells cultured from neonatal hemangiomas. Am. J. Pathol. 150:1315-1326.

6. Virchow, R. 1863. Die Krankhaften Geschwulste. August Hirschwald. Berlin, Germany. 306 pp.

7. Pack, G., and Miller, T.R. 1950. Hemangiomas; classification, diagnosis, and treatment. Angiology. 1:405-426.

8. Malan, E. 1974. Vascular malformations (angiodysplasias). In Carlo Erba Foundation. Milan, Italy. 4.

9. Smoller, B.R., and Apfelberg, D.B. 1993. Infantile (juvenile) capillary hemangioma: a tumor of heterogeneous cellular elements. J. Cutan. Pathol. 20:330-336.

10. Dosanjh, A., et al. 2000. In vitro characteristics of neonatal hemangioma endothelial cells: similarities and differences between normal neonatal and fetal endothelial cells. J. Cutan. Pathol. 27:441-450.

11. Shmelkov, S.V., St. Clair, R., Lyden, D., and Rafii, S. 2005. AC133/CD133/Prominin-1. Int. J. Biochem. Cell Biol. 37:715-719.

12. Yu, Y., Flint, A.F., Mulliken, J.B., Wu, J.K., and Bischoff, J. 2004. Endothelial progenitor cells in infantile hemangioma. Blood. 103:1373-1375.

13. Khan, Z.A., et al. 2006. Endothelial progenitor cells from infantile hemangioma and umbilical cord blood display unique cellular responses to endostatin. Blood. 108:915-921.

14. Bauland, C.G., et al. 2006. The pathogenesis of hemangiomas: a review. Plast. Reconstr. Surg. 117:29e-35e.

15. Wu, X., et al. 2004. Tissue-engineered microvessels on three-dimensional biodegradable scaffolds using human endothelial progenitor cells. Am.J. Physiol. Heart Circ. Physiol. 287:H480-H487.

16. O’Brien, C.A., Pollett, A., Gallinger, S., and Dick, J.E. 2007. A human colon cancer cell capable of initiating tumour growth in immunodeficient mice. Nature. 445:106-110.

17. Ricci-Vitiani, L., et al. 2007. Identification and expansion of human colon-cancer-initiating cells. Nature. 445:111-115.

18. Melero-Martin, J.M., et al. 2007. In vivo vasculogenic potential of human blood-derived endothelial progenitor cells. Blood. 109:4761-4768.

19. Baal, N., et al. 2004. Expression of transcription factor Oct-4 and other embryonic genes in CD133 positive cells from human umbilical cord blood. Thromb. Haemost. 92:767-775.

20. Okuda, T., van Deursen, J., Hiebert, S.W., Grosveld, G., and Downing, J.R. 1996. AML1, the target of multiple chromosomal translocations in human leukemia, is essential for normal fetal liver hematopoiesis. Cell. 84:321-330.

21. Li, Z., Chen, M.J., Stacy, T., and Speck, N.A. 2006. Runx1 function in hematopoiesis is required in cells that express Tek. Blood. 107:106-110.

22. Reyes, M., et al. 2002. Origin of endothelial progenitors in human postnatal bone marrow. J. Clin. Invest. 109:337-346.

23. Jiang, Y., et al. 2002. Pluripotency of mesenchymal stem cells derived from adult marrow. Nature. 418:41-49.

24. Egusa, H., Schweizer, F.E., Wang, C.C., Matsuka, Y., and Nishimura, I. 2005. Neuronal differentiation of bone marrow-derived stromal stem cells involves suppression of discordant phenotypes through gene silencing. J. Biol. Chem. 280:23691-23697.

25. Tondreau, T., et al. 2004. Bone marrow-derived mesenchymal stem cells already express specific neural proteins before any differentiation. Differentiation. 72:319-326.

26. Pittenger, M.F., et al. 1999. Multilineage potential of adult human mesenchymal stem cells. Science. 284:143-147.

27. Ritter, M.R., Reinisch, J., Friedlander, S.F., and Friedlander, M. 2006. Myeloid cells in infantile hemangioma. Am. J. Pathol. 168:621-628.

28. Choi, K., Kennedy, M., Kazarov, A., Papadimitriou, J.C., and Keller, G. 1998. A common precursor for hematopoietic and endothelial cells. Development. 125:725-732.

29. Ito, Y., Iwamoto, Y., Tanaka, K., Okuyama, K., and Sugioka, Y. 1996. A quantitative assay using basement membrane extracts to study tumor angiogenesis in vivo. Int. J. Cancer. 67:148-152.

30. Hoang, M.V., and Senger, D.R. 2005. In vivo and in vitro models of Mammalian angiogenesis. Methods Mol. Biol. 294:269-285.

31. Yu, Y., et al. 2006. Mesenchymal stem cells and adipogenesis in hemangioma involution. Stem Cells. 24:1605-1612.

32. Parums, D.V., et al. 1990. JC70: a new monoclonal antibody that detects vascular endothelium associated antigen on routinely processed tissue sections. J. Clin. Pathol. 43:752-757.
33. North, P.E., Waner, M., Mizeracki, A., and Mihm, M.C., Jr. 2000. GLUT1: a newly discovered immunohistochemical marker for juvenile hemangiomas. Hum. Pathol. 31:11-22.

34. North, P.E., et al. 2001. A unique microvascular phenotype shared by juvenile hemangiomas and human placenta. Arch. Dermatol. 137:559-570.

35. Kawaguchi, N., et al. 1998. De novo adipogenesis in mice at the site of injection of basement membrane and basic fibroblast growth factor. Proc. Natl. Acad. Sci. U.S. A. 95:1062-1066.

36. Kimura, Y., Ozeki, M., Inamoto, T., and Tabata, Y. 2002. Time course of de novo adipogenesis in matrigel by gelatin microspheres incorporating basic fibroblast growth factor. Tissue Eng. 8:603-613.

37. Tabata, Y., et al. 2000. De novo formation of adipose tissue by controlled release of basic fibroblast growth factor. Tissue Eng. 6:279-289.

38. Greenberg, A.S., et al. 1991. Perilipin, a major hormonally regulated adipocyte-specific phosphoprotein associated with the periphery of lipid storage droplets. J. Biol. Chem. 266:11341-11346.

39. Singh, S.K., et al. 2003. Identification of a cancer stem cell in human brain tumors. Cancer Res. 63:5821-5828.

40. Salmaggi, A., et al. 2006. Glioblastoma-derived tumorospheres identify a population of tumor stemlike cells with angiogenic potential and enhanced multidrug resistance phenotype. Glia. 54:850-860.

41. Collins, A.T., Berry, P.A., Hyde, C., Stower, M.J., and Maitland, N.J. 2005. Prospective identification of tumorigenic prostate cancer stem cells. Cancer Res. 65:10946-10951.

42. Richardson, G.D., et al. 2004. CD133, a novel marker for human prostatic epithelial stem cells. J. Cell Sci. 117:3539-3545.

43. Obeso, J., Weber, J., and Auerbach, R. 1990. A hemangioendothelioma-derived cell line: its use as a model for the study of endothelial cell biology. Lab. Invest. 63:259-269.

44. Bautch, V.L., Toda, S., Hassell, J.A., and Hanahan, D. 1987. Endothelial cell tumors develop in transgenic mice carrying polyoma virus middle $\mathrm{T}$ oncogene. Cell. 51:529-537.

45. Tang, Y., et al. 2007. A novel in vivo model of human hemangioma: xenograft of human hemangioma tissue on nude mice. Plast. Reconstr. Surg. 120:869-878.

46. Barnes, C.M., et al. 2005. Evidence by molecular profiling for a placental origin of infantile hemangioma. Proc. Natl. Acad. Sci. U. S. A. 102:19097-19102.

47. Tai, M.H., et al. 2005. Oct4 expression in adult human stem cells: evidence in support of the stem cell theory of carcinogenesis. Carcinogenesis. 26:495-502.

48. Choi, S.J., et al. 2003. AML-1A and AML-1B regulation of MIP-1alpha expression in multiple myeloma. Blood. 101:3778-3783. 